\title{
HADRON STAR MODELS
}

\author{
JEFFREY M. COHEN* and GERHARD BÖRNER** \\ High Energy Astrophysics Laboratory, Goddard Space Flight Center, Greenbelt, Md., U.S.A.
}

\begin{abstract}
The properties of fully relativistic rotating hadron star models are discussed using models based on recently developed equations of state. All of these stable neutron star models are bound with binding energies as high as $\sim 25 \%$. During hadron star formation, much of this energy will be released. The consequences, resulting from the release of this energy, are examined.
\end{abstract}

\section{Introduction}

In a number of the preceding papers of this symposium, the nuclear physics of cold dense matter was discussed and equations of state (pressure $p$ vs. density $\varrho$ ) of the form $p=p(\varrho)$ obtained. Since the Fermi levels involved are much greater than $k T$ for neutron stars accepted as typical pulsar models, this approximation is reasonable. Figure 1 as a flow chart of the procedure leading to observations. As can be seen from the top line (and is generally known), the use of the complete set of Einstein equations (10 non-linear simultaneous partial differential equations) have led to no neutron star models. No one has solved this complete set of equations on the computer. As the arrows indicate, all attempts to do this have led nowhere. Contemplation of Figure 1 will take the place of further discussion along this line.

A more successful approach was used by Oppenheimer and Volkoff (1939) using results of Tolman (1939). They used spherical symmetry to reduce Einstein's equations to a simple form. Generally denoted by TOV, the equations they used are obtained by eliminating $\phi$ from the following set

$$
\begin{aligned}
\partial_{r} m & =4 \pi r^{2} \varrho \\
c^{2} \partial_{r} \phi & =G\left(m+4 \pi r^{3} \mathrm{pc}^{-2}\right) r^{-1}\left(r-2 G M c^{-2}\right)^{-1} \\
\partial_{r} p & =-\left(\varrho+\mathrm{pc}^{-2}\right) c^{2} \partial_{r} \phi .
\end{aligned}
$$

We find the above set more useful, however, since it gives the red shift $Z=e^{-\phi_{-}}-1$ automatically. In these equations $m$ is the gravitational mass, $\phi$ is a relativistic generalization of the Newtonian gravitational potential (it is equal to the Newtonian value in the weak field limit), and $r$ is a radial coordinate chosen in such a way that the surface area of a sphere is $4 \pi r^{2}$ (Cohen and Cohen, 1969). A subscript $r$ denotes differentiation with respect to $r$.

The equations were integrated numerically by Volkoff on a hand calculator using an equation of state for a free Fermi gas of neutrons. Neutron decay was neglected since weak interactions as well as strong interactions were neglected. These models of

* Permanent address: Physics Department, University of Pennsylvania, Philadelphia, Pa. 19104. National Academy of Science - NRC Senior Research Associate.

** Permanent address: Max-Planck Institut für Physik and Astrophysik, München, Germany. National Academy of Science - NRC Research Associate. 
Oppenheimer and Volkoff (1939) gave rise to the name neutron star, as has been mentioned in previous papers, but the inclusion of strong and weak interactions gives rise to models composed of many varieties of hadrons. For this reason, the more precise name hadron star has been utilized. The most massive neutron star models obtained by Oppenheimer and Volkoff (1939) had a mass of $\sim 0.72 m_{\odot}$. These models are non-rotating (Figure 1) and since pulsars are presently believed to be rotating neutron stars, further information is needed.

As will be seen below, typical neutron stars have a radius only a few times their gravitational radius. Thus, general relativistic effects are quite important and must be taken into account. One such general relativistic effect is the dragging along of inertial frames by rotating bodies (Brill and Cohen, 1966). Unlike Newtonian mechanics, in which a gyroscope points towards the same distant star independent of the motion of nearby masses, rotating masses in general relativity drag along the inertial frames and cause the rotation axis of a gyroscope to precess.

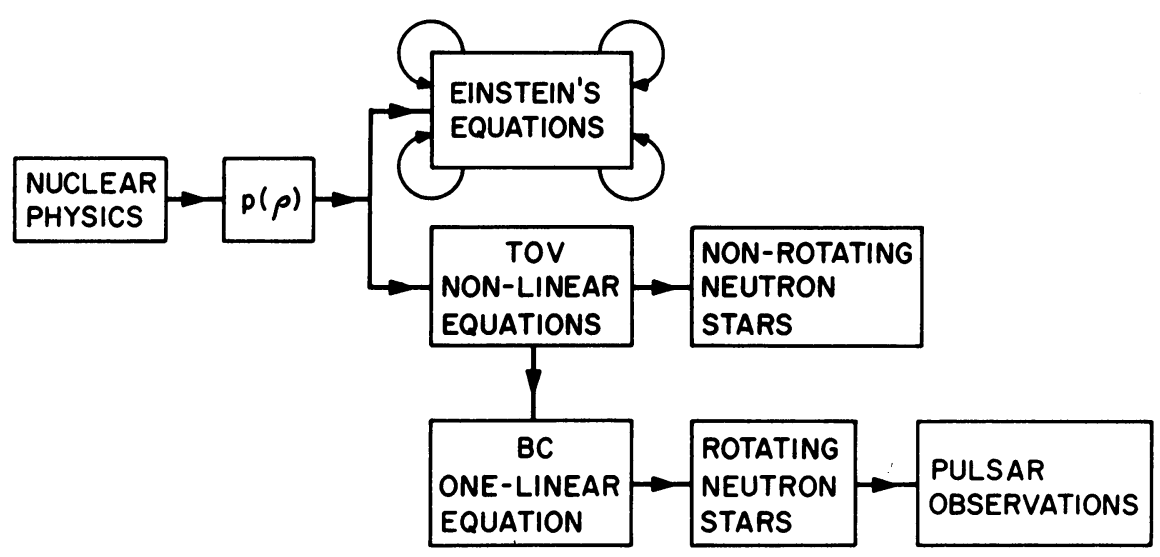

Fig. 1. Flow chart of the procedure leading from nuclear physics to astrophysical observations.

A complete general relativistic description of rotating neutron stars consists of solving the full set of Einstein equations. In order to progress further than the top line of Figure 1, another method was developed. This method (Brill and Cohen, 1966), valid for slowly rotating stars, requires the solution of only one linear equation once Equations (1) are integrated. This method is useful for treating pulsars since even the Crab pulsar can be considered a slowly rotating object in the sense that the velocity of any element of the star is small compared to the light velocity and the centrifugal force is small compared to the gravitational force.

The equation to be solved to treat rotating neutron stars is (Brill and Cohen, 1966; Cohen and Brill, 1968)

$$
\left[A^{-1} B^{-1} r^{4} \Omega_{r}\right]_{r}=-16 \pi B A^{-1}\left(\varrho+\mathrm{pc}^{-2}\right)(\omega-\Omega) G c^{-2} .
$$

The quantity $\Omega$ is the angular velocity of inertial frames along the rotation axis where 
it can be measured by observing the precession rate of the axis of a gyroscope and $\omega$ is the angular velocity of the star. Except for $\Omega$, all quantities are known from (1) or are given. To simplify the equations we have used the quantities $A$ and $B$ defined as

$$
A=e^{\phi}, \quad B^{-2}=1-2 G m r^{-1} c^{-2} .
$$

As boundary conditions on $\Omega$ we have $\Omega \sim$ constant near the origin and $\Omega \sim r^{-3}$ outside the star.

Once $\Omega$ is determined, it is straightforward to compute quantities of astrophysical interest such as the angular momentum $J$ and the rotational energy $E_{\text {rot }}$. The fully relativistic expression for the moment of inertia of a uniformly rotating body is (Cohen, 1970; Cohen, 1972; and Cohen and Cameron, 1971)

$$
I=(8 \pi / 3) \int_{R_{1}} \varrho r^{4}\left[\left(1+p \varrho^{-1} c^{-2}\right) B A^{-1}\left(1-\Omega \omega^{-1}\right)\right] \mathrm{d} r
$$

This expression differs from the corresponding Newtonian one by the quantity in brackets. Note that the pressure as well as the density contributes. Also the motion of inertial frames $\Omega$, the red shift $\left(Z=e^{-\phi}-1=A^{-1}-1\right)$ and space curvature enter into this general relativistic expression. Use will be made of (2) and (3) once non-rotating models have been discussed using (1).

\section{Properties of Hadron Star Models}

Numerical integration of (1) gives the parameters of neutron star models (more recently called hadron star models) for various equations of state. Figure 2 shows the variation of the gravitational mass with density. As expected from their very soft equation of state, the neutron star models of Leung and Wang (1971) have very low mass. The maximum mass of their models is not only lower than any of the others shown, but it is also lower than that of Oppenheimer and Volkoff (1939) who neglected repulsion due to strong interactions. If Leung and Wang are excluded from the graph, then agreement between the remaining curves is quite reasonable. Each of these remaining curves CCLR (Cohen et al., 1970), BJ (Bethe and Johnson, 1973), BBS (Bethe et al., 1970), and BPS (Baym et al., 1971) give a maximum mass higher than that of a free Fermi gas. Such behavior is not surprising because of the repulsive core of hadron interactions.

The CCLR equation of state was obtained using the Levinger-Simons velocity dependent potentials while the later calculations BBS, BPS, BJ used the Reid soft core potential. In the high density region above $\sim 2 \times 10^{14} \mathrm{~g} \mathrm{~cm}^{-3}$, use was made of the work of Pandharipande's pure neutron results by BBS and his hyperon results by BPS.

Figure 3 gives the density distribution of selected neutron star models. Note the kink in the curves at $\varrho_{k} \sim 10^{11} \mathrm{~g} \mathrm{~cm}^{-3}$. This is the point where nuclei become unstable against the emission of neutrons; such neutron drip (Harrison et al., 1965) causes the 


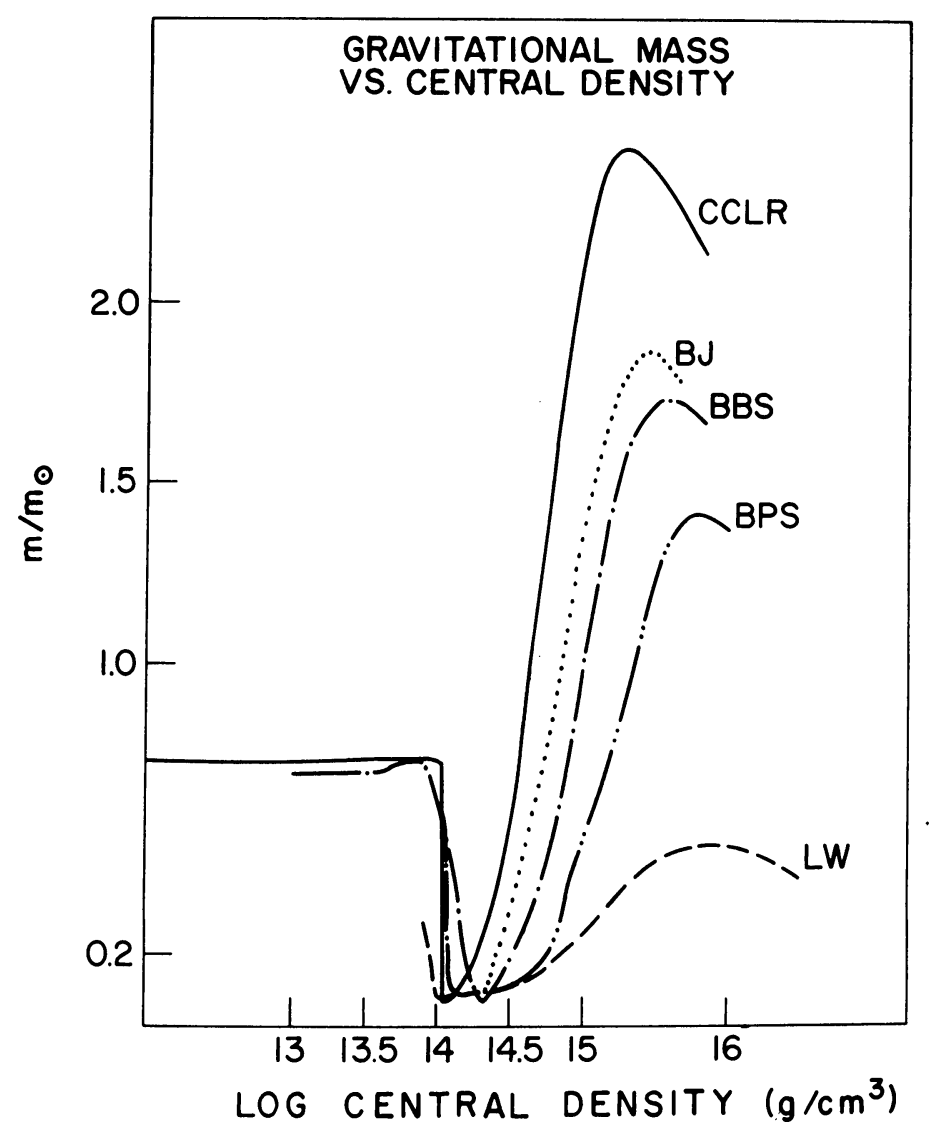

Fig. 2. Gravitational mass vs density for various equations of state.

equation of state to be quite soft in the density region from just above this density $\varrho_{k}$ to the point where the neutron concentration is sufficient to give a sizeable pressure from the degenerate neutrons. From a plot of the adiabatic index $\Gamma$ vs. $\varrho$ (Cohen and Cameron, 1971), this effect manifests itself as a rapid decrease in $\Gamma$ at $\sim 3 \times 10^{11} \mathrm{~g} \mathrm{~cm}^{-3}$.

The angular velocity of inertial frames along the rotation axis as a function of radius is depicted in Figures 4, 5, and 6 for three different equations of state - CCLR, BJ, BBS. The curves are almost identical even though the underlying equations of state are based on rather different assumptions and potentials; a repulsive core is their common feature. Note that, near the center of the uniformly rotating neutron star, the inertial frames can rotate with angular velocity $\sim 70 \%$ that of the star, dropping to $\sim 30 \%$ near the surface.

Such large dragging of inertial frames makes a significant contribution to the moment of inertia as can be seen from (3). The moment of inertia is plotted in Figure 7 for various equations of state. An interesting property of the CCLR, BJ, and BBS curves is that the moment of inertia peaks at densities below the mass peak. Hence 


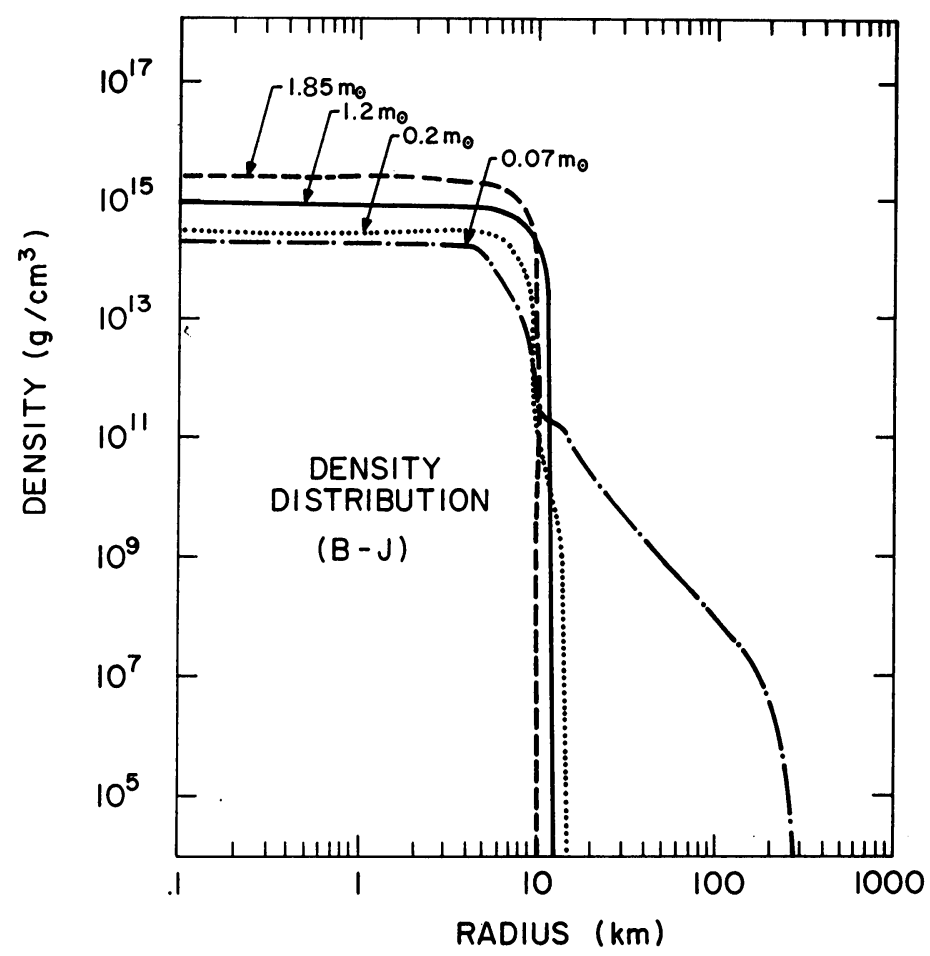

Fig. 3. Variation of density with stellar radius for a selected model based on the BBS equation of state.

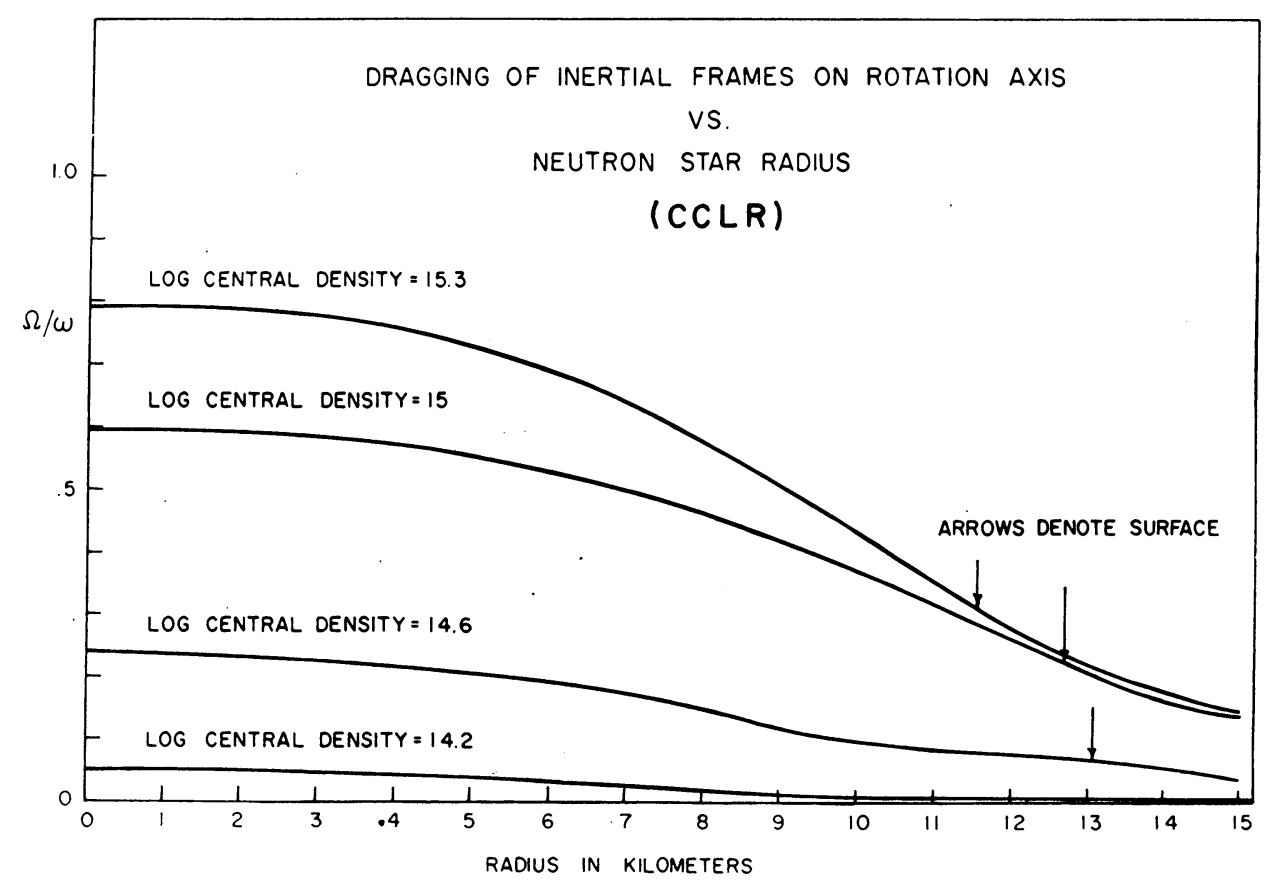

Fig. 4. Angular velocity of inertial frames as a function of radius for models based on equation of state of Cohen et al. (1970). 


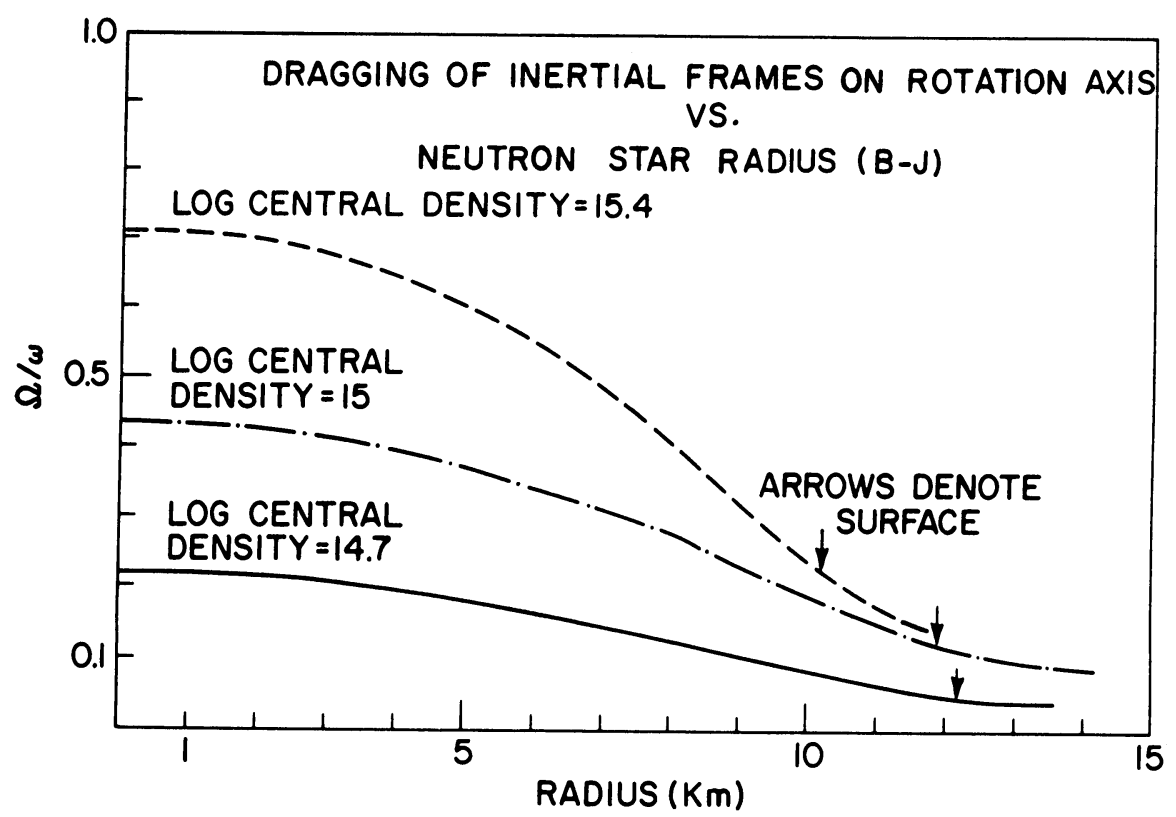

Fig. 5. Angular velocity of inertial frames as a function of radius for models based on equation of state of Bethe and Johnson.

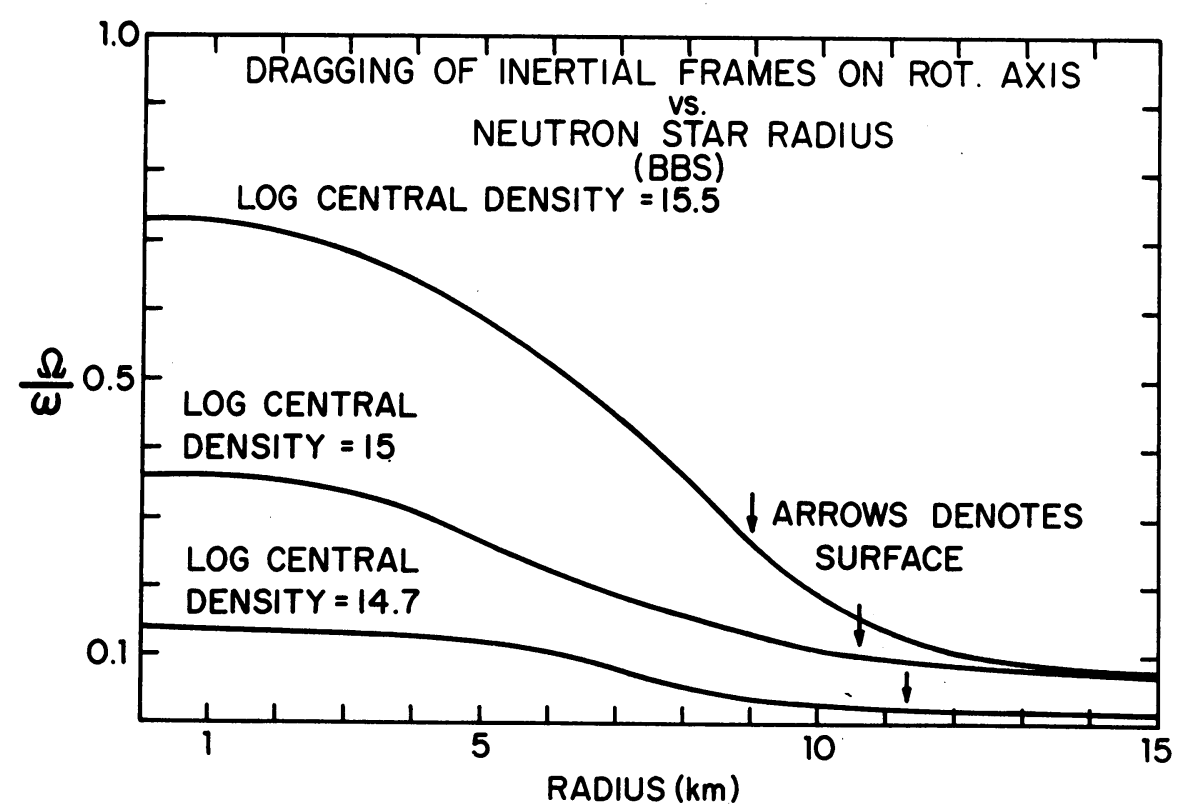

Fig. 6. Angular velocity of inertial frames as a function of radius for models based on equation of state of Bethe et al. (1970). 
the addition of material to massive (stable) neutron stars can reduce the moment of inertia - a property which does not depend on a particular equation of state.

The horizontal lines $a$ and $b$ represent lower limits on the moment of inertia which can be obtained from comparison with observation. Further discussion of these lines will not be given here since a detailed discussion of all the horizontal lines appears in the next paper.

From the density distribution in Figure 3, it can be seen that the relative size of the star's outer crystalline region decreases as the star becomes more massive. Thus it is not surprising that, with increasing central density, there is a decrease in the ratio of the crust's moment of inertia to that of the entire star, as can be seen in Figure 8. We have assumed that the star's crust extends up to densities of $\sim 2 \times 10^{14} \mathrm{~g} \mathrm{~cm}^{-3}$ (Baym et al., 1971).

Now that the properties of neutron star models have been determined, it is of interest to consider the question of how neutron stars are formed (Börner et al., 1973).

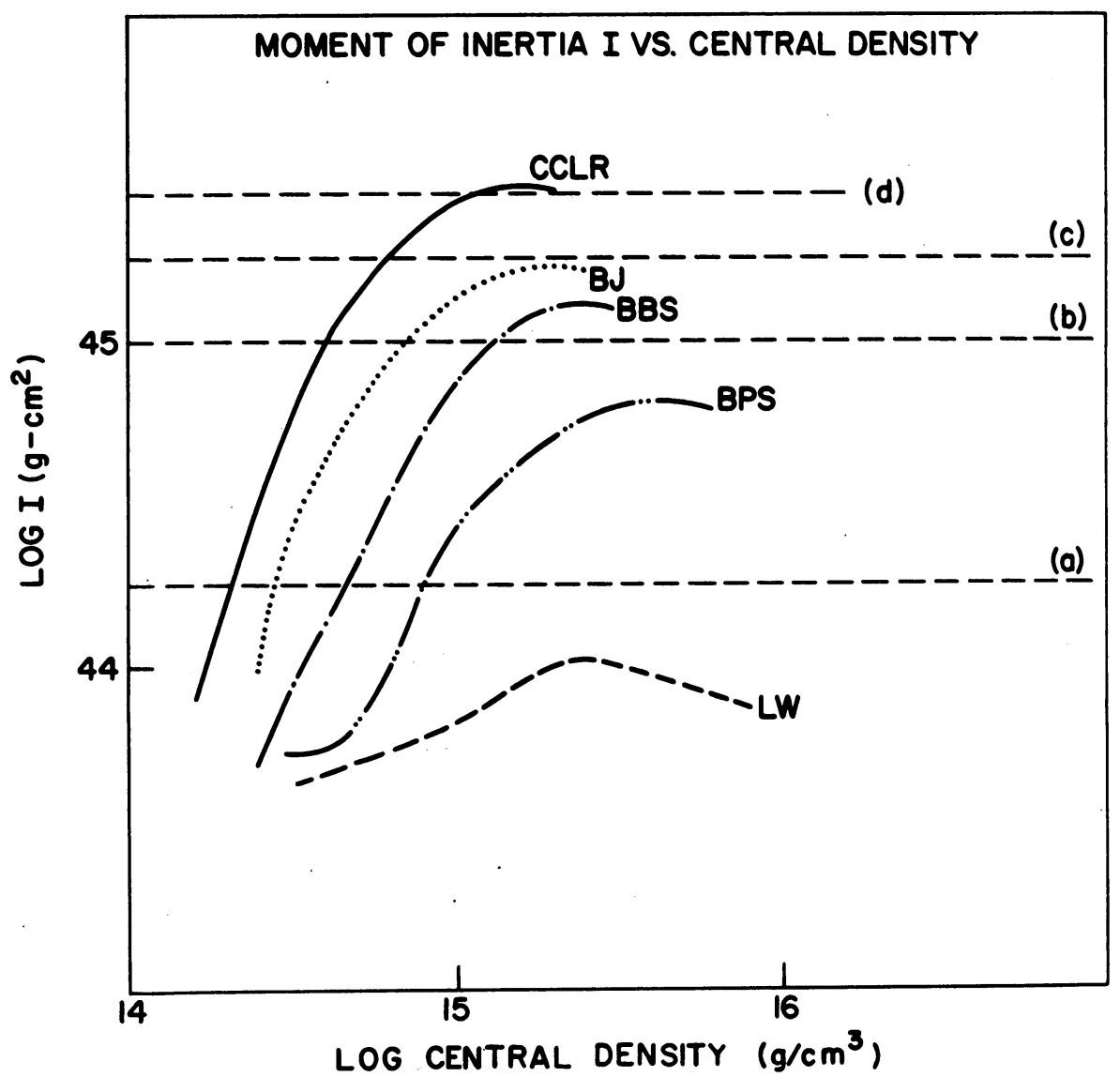

Fig. 7. Moment of inertia (curved lines) of neutron star models as a function of density for various equations of state. The value plotted includes general relativistic effects such as dragging of inertial frames, gravitational red shift, and the contribution from the pressure. 


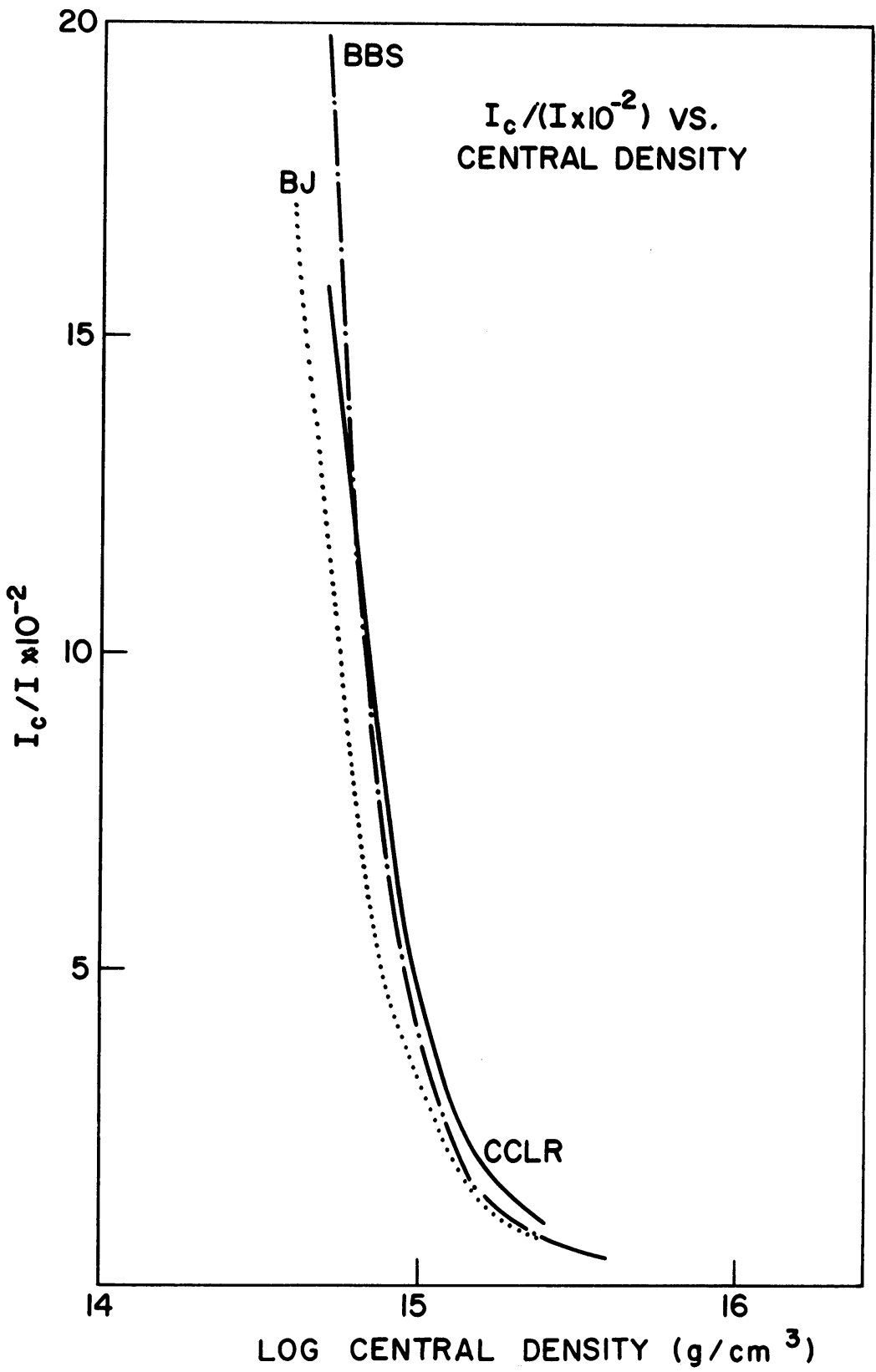

Fig. 8. Ratio of the crust moment of inertia to the moment of inertia of the entire star as a function of central density. 


\section{Hadron Star Formation}

The endpoint of the stellar evolution of stars with mass less than $\sim 1.2 m_{\odot}$ are believed to be white dwarfs (e.g., Chandrasekhar, 1939). It has been suggested (Paczynski and Ziolkowski, 1967; Lucy, 1967; Roxburgh, 1967) that stars in the mass range $\sim 1.2 \mathrm{~m}_{\odot}$ and $\sim 4 m_{\odot}$ develop dilute envelopes with extensive ionization regions at low gravitational potential. They predict that recombination produces an unbound envelope which is ejected leaving a white dwarf possibly surrounded by a planetary nebula (Finzi and Wolf, 1969).

In the mass range $\sim 4 m_{\odot}$ to $9 m_{\odot}$ evolutionary calculations have given models which develop a degenerate core with mass $\sim 1.4 m_{\odot}$ (Rose, 1969; Arnett, 1969; Paczynski, 1970). Although most of the mass in these models is contained in a large dilute envelope, the core's structure is essentially independent of the model's total mass (Paczynski, 1970; Barkat, 1971). The core is degenerate and its structure is essentially that of a white dwarf. As with a white dwarf, if the core's mass exceeds the Chandrasekhar limit, gravitational collapse will ensue.

Since $\mathrm{C}^{12}$ has a high electron capture threshold, pure $\mathrm{C}^{12}$ white dwarfs undergo general relativistic instability at a central density $\left(\sim 3 \times 10^{10} \mathrm{~g} \mathrm{~cm}^{-3}\right)$ below that where electron capture sets in (Cohen et al., 1969). Stars with more realistic composition become unstable at lower densities $\sim 3$ to $9 \times 10^{9} \mathrm{~g} \mathrm{~cm}^{-3}$ because of electron capture (Cohen et al., 1969; Colgate, 1971; Wheeler et al., 1970; Barkat et al., 1970, 1971). From the above it seems reasonable to expect the formation of a collapsed remnant - neutron star or black hole (collapsar) - in the evolution of sufficiently massive stars. Since pulsars are generally believed to be rotating neutron stars (Gold, 1968) it seems likely that a neutron star remnant will be the endpoint of stellar evolution in at least some cases.

If $\mathrm{C}^{\mathbf{1 2}}$ ignition occurs at densities lower than that where collapse sets in, Arnett (1969) has suggested that the entire core will detonate and the star will be completely disrupted. Later, Barkat et al. (1972) have cast serious doubt on the claim that carbon ignition in this mass range must lead to total disruption of the star. This was done by taking into account more detailed neutrino loss mechanisms. But they neglected URCA processes which, they suggest, may further reduce the possibility of disruption. Paczynski (1972) has suggested that $C^{12}$ ignition causes the core to become convective. Such a convective core can emit a large neutrino flux via the URCA process. Matter from high density regions moves to regions of lower density where it $\beta$-decays emitting neutrinos in the process. Similarly, lower density matter in the core moves to higher density regions and through electron capture also emits neutrinos. The neutrino flux from such a convective region (if it exists) could remove the energy generated by carbon burning and thwart the disruptive tendencies of $\mathbf{C}^{\mathbf{1 2}}$ ignition. Detailed convective core calculations by Barkat (1973) are in progress.

Recent measurements by Mazarakis and Stevens (1972) indicate a rapid decrease in $\mathrm{C}^{12}-\mathrm{C}^{12}$ reaction cross section at low energies. Thus, a small decrease in temperature (e.g., from neutrino losses) will give a relatively large decrease in the energy generated from the $\mathrm{C}^{12}-\mathrm{C}^{12}$ reaction. 
Recently Gordon (Pecker-Wimel) (1972a, b) has studied the physical conditions existing in a supernova shell using observations of type I supernovae spectra. By considering emission and absorption lines from the expanding shell, she found that a continuous source of heating after the explosion is required by the observation. She suggests a neutron star remnant emitting charged particles and/or low frequency waves as a consistent explanation.

Further discussion of the situation can be found in Börner et al. (1973). A resolution of this controversy will not be attempted here. Rather we assume the formation of a neutron star remnant and examine the consequences.

\section{Hadron Star Formation Energy}

As mentioned above, if the degenerate core's mass exceeds the Chandrasekhar limit for white dwarfs, the core becomes unstable against gravitational collapse. If the core's collapse leads to neutron star formation and the ejection of the outer envelope, the energy released will be the difference between the binding energy of the neutron star plus that of the ejected envelope and any ejected portion of the white dwarf core minus the binding energy of the presupernova red giant. The red giant binding energy includes contributions from the envelope, nuclear binding, gravitational binding, and the kinetic energy of the degenerate electrons. Consideration of this energy balance shows that neutron stars can be formed only if there is a net positive energy release.

If a degenerate core of mass $\sim 1.4 m_{\odot}$ - just above the Chandrasekhar limit collapses to form a neutron star and little mass is lost (the idea of a small mass loss is suggested by consideration of the energy balance of the Crab and Vela nebulae by Börner and Cohen (1972)), then the resulting neutron star will have a mass $\sim 1.2 m_{\odot}$. The mass difference $\sim 0.15 \mathrm{~m}_{\odot}$ is released as an energy of $\sim 3 \times 10^{53} \mathrm{erg}$. Nowhere else in physics or astrophysics has there been proposed a mechanism which gives such a large energy release as this gravity bomb. Neutron stars with mass above $\sim 1.2 \mathrm{~m}_{\odot}$ have binding energies in the range $\sim 10$ to $25 \%$ while thermonuclear fusion reactions release as energy only $\sim 0.7 \%$ of the rest mass. Thus the efficiency of the gravity bomb is much higher than that of thermonuclear devices. Luckily, however, the problem of delivering a $2 \times 10^{33} \mathrm{~g}$ device seems to be rather difficult.

A possible sequence of events leading to neutron star formation may be the following: The degenerate core collapses from the radius $\sim 10^{8} \mathrm{~cm}$ of a dense white dwarf near the mass peak $\sim 1.4 m_{\odot}$ (Cohen et al., 1969; Barkat et al., 1971) to a neutron star of radius $\sim 10^{6} \mathrm{~cm}$. The core collapses so rapidly that the neutron star is formed before much of the envelope can follow. As the core collapses, binding energy will be released as heat resulting in the cavity being filled with black-body radiation and relativistic particles in thermal equilibrium with electron-positron pairs.

As mentioned above, if little or no mass is lost from, or accreted on, the core during collapse, the resulting neutron star will have a gravitational mass of $\sim 1.2 \mathrm{~m}_{\odot}$ and released binding energy $\sim 3 \times 10^{53} \mathrm{erg}$. In a cavity of radius $10^{8} \mathrm{~cm}$, this energy will give rise to a black-body temperature of $\sim 4.4 \times 10^{10} \mathrm{~K}$. Since $k T \sim 3.8 \mathrm{MeV}$ is above 
the electron-positron pair production threshold, the cavity will contain relativistic positrons and electrons as well as photons. The electron-positron pair energy is $7 / 4$ the photon energy. The above temperature includes the effect of pair production; neglect of this effect would have given a higher value.

An apparent difficulty is that the black-body curve peaks at a frequency $\sim 10^{21} \mathrm{~Hz}$ which is above the outer shell's plasma frequency of $\lesssim 10^{19} \mathrm{~Hz}$. Because of this, one might think that the radiation will pass through the outer envelope. But this is not the case. The range of electrons and positrons is reduced by collision losses to about $1 \mathrm{~g} \mathrm{~cm}^{-2}$ which gives a mean free path of $\sim 100 \mathrm{~cm}$, much less than the envelope thickness $\sim 10^{12} \mathrm{~cm}$. Similarly, the photon component is also stopped very efficiently at the inner edge of the envelope by Compton scattering, pair production, and freefree absorption. Some energy loss is expected from other channels (neutrinos and gravitational radiation) but this loss is expected to be small. The loss through these channels is somewhat uncertain but recent neutrino loss estimates are all of the same order $\sim 1$ to $4 \times 10^{52} \mathrm{erg}$. Since this is small compared to the total binding energy released, it will have a negligible effect on the energy balance. But such a neutrino luminosity is a thousand times the optical luminosity of supernovae. It may be possible to observe these neutrinos with recently developed apparatus (Bozoki and Lande, 1973). The total energy emitted as gravitational waves cannot exceed the energy stored in pulsation $\left(\sim 5 \times 10^{52} \mathrm{erg}\right)$ and rotation $\left(\sim 5 \times 10^{52} \mathrm{erg}\right)$. This is an upper limit on the gravitational radiation emitted; we expect the actual value to be much less. If pulsars are rotating objects, then at least some of the rotational energy remains after the supernova explosion. Recall that there are no gravitational waves emitted during spherically symmetric collapse. Thus, we expect most of the binding energy to be deposited in black-body radiation and relativistic particles trapped within the shell.

The pressure on the outer envelope exerted by such a trapped relativistic gas will be much higher than that exerted by the white dwarf core prior to collapse. Consequently, the envelope will be pushed out quite efficiently if the gas energy is greater than the envelope's potential energy.

The outer envelope's potential energy is given by

$$
P E=4 \pi G \int_{R_{2}}^{R_{4}} \varrho m(r) r \mathrm{~d} r,
$$

where $R_{1} \geqslant 10^{8} \mathrm{~cm}$ is the inner radius (discussed above), and $R_{2} \cong 10^{12} \mathrm{~cm}$ is the envelope's outer radius. By choosing. $n$ larger than values obtained from detailed computer models (Schwarzschild, 1957), an upper limit on the potential energy is obtainable by assuming a density profile $\varrho=\alpha r^{-n}$ with constants $\alpha$ and $n$. If $0 \leqslant n \leqslant 2.4$ (a typical value for low mass red giants is $n=1.9$; Schwarzschild (1957)), we find that there is sufficient energy to blow off the envelope of red giants with mass in excess of $500 m_{\odot}$. Here we assumed that these massive red giants develop dense cores in the late stages of their evolution as do the lower mass stars and that the core mass is less than $\sim 2.5 m_{\odot}$. Thus, it is energetically possible for even the most massive stars observed 


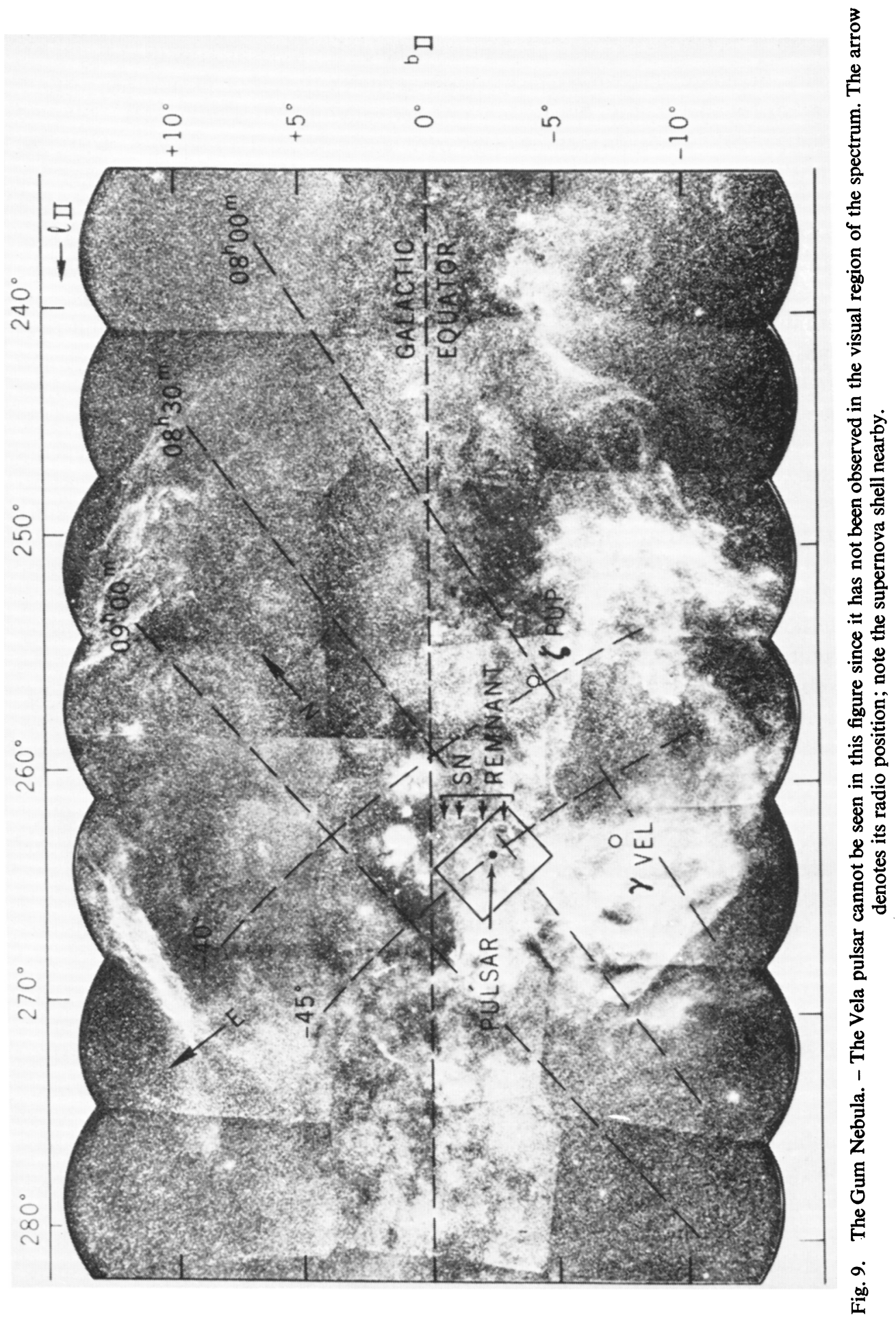


( $m<70 m_{\odot}$, which is comparable to Chandrasekhar's limit for red giants; see also Larson and Starrfield (1971)) to leave a neutron star as a remnant of a supernova explosion.

It has been asserted (Harrison et al., 1965) that once they have reached the end point of their thermonuclear evolution, stars with mass exceeding the maximum mass for stable neutron stars $\left(\sim 2 m_{\odot}\right)$ cannot escape from collapse into a black hole. Our results indicate that even very massive stars do not necessarily collapse into a singularity, but can leave a stable neutron star as a remnant. This suggests that black holes, if they exist, may not be formed during supernova explosion. A possible mechanism for black hole formation is discussed elsewhere (Börner et al., 1973).

\section{Ionization of the Gum Nebula}

In Figure 9 is shown a region of the sky known as the Gum nebula. For an extensive discussion of this region see, for example, Maran et al. (1971). The properties of primary interest here are its size $\sim 460 \mathrm{pc}$, age $\sim 10^{4} \mathrm{yr}$ and the number of ionized particles it contains $\sim 2 \times 10^{62}$. Note that near the center of the Gum nebula is the Vela pulsar and supernova remnant. Here we will consider the possibility that the Vela supernova ionized the Gum nebula. To ionize interstellar hydrogen by collisions with protons with energy $\gtrsim 0.1 \mathrm{MeV}$, an energy loss of about $36 \mathrm{eV}$ per ion pair is required (Bethe and Ashkin, 1953). This implies that a total energy of $\sim 10^{52} \mathrm{erg}$ was necessary to ionize the entire region (Ramaty and Boldt, 1971).

If the initial presupernova red giant has a mass $\sim 15 m_{\odot}$ and $3 \times 10^{53} \mathrm{erg}$ was imparted to the outer envelope, then the average energy per baryon will be $\sim 10 \mathrm{MeV}$. The velocity of $10 \mathrm{MeV}$ protons is $\sim 4 \times 10^{9} \mathrm{~cm} \mathrm{~s}^{-1}$. In $10^{4} \mathrm{yr}$, these protons willcover a distance of $\sim 400 \mathrm{pc}$-comparable to the observed size of the Gum nebula. Also the ionization efficiency (Ramaty and Boldt, 1971) by $10 \mathrm{MeV}$ protons is such that $\sim 2 \times 10^{52} \mathrm{erg}$ is imparted to the nebulae in this time.

\section{References}

Arnett, W. D.: 1969, Astrophys. Space Sci. 5, 180.

Barkat, Z.: 1971, Astrophys. J. 163, 433.

Barkat, Z.: 1973 (to be published).

Barkat, Z., Buchler, J. R., and Wheeler, J. C.: 1970, Astrophys. Letters 6, 117.

Barkat, Z., Buchler, J. R., and Wheeler, J. C.: 1971, Astrophys. J. 8, 21.

Barkat, Z., Wheeler, J. C., and Buchler, J. C.: 1972, Astrophys. J. 171, 651.

Baym, G., Pethick, C., and Sutherland, P.: 1971, Astrophys. J. 170, 299.

Bethe, H. A. and Ashkin, J.: 1953, in E. Serge (ed.), Experimental Nuclear Physics 1, 233. John Wiley and Sons Inc., N.Y.

Bethe, H. A. and Johnson, M.: 1973 (to be published).

Bethe, H. A., Börner, G., and Sato, K.: 1970, Astron. Astrophys. 7, 279.

Börner, G. and Cohen, J. M.: 1972, Astron. Astrophys. 19, 109.

Börner, G., Cohen, J. M., and Ramaty, R.: 1973 (to be published).

Bozoki, G. and Lande, K.: 1973 (to be published).

Brill, D. R. and Cohen, J. M.: 1966, Phys. Rev. 143, 1011.

Chandrasekhar, S.: 1939, Introduction to the Study of Stellar Structure, Dover Press, N. Y.

Cohen, J. M.: 1970, Astrophys. Space Sci. 6, 263. 
Cohen, J. M.: 1972, General Relativity and Gravitation Res. 3, 221.

Cohen, J. M. and Brill, D. R.: 1968, Nuovo Cimento 50B, 209.

Cohen, J. M. and Cameron, A. G. W.: 1971, Astrophys. Space Sci. 10, 227.

Cohen, J. M. and Cohen, M. D.: 1969, Nuovo Cimento 60B, 241.

Cohen, J. M., Langer, W.D., Rosen, L. C., and Cameron, A. G. W.: 1970, Astrophys. Space Sci. 6, 228.

Cohen, J. M., Lapidus, A., and Cameron, A. G. W.: 1969, Astrophys. Space Sci. 5, 113.

Colgate, S. A.: 1971, Astrophys. J. 163, 221.

Finzi, A. and Wolf, R. A.: 1969, Astrophys. J. Letters 155, L107.

Gold, T.: 1968, Nature 218, 731.

Gordon (Pecker-Wimel), C.: 1972a, Astron. Astrophys. 20, 79.

Gordon (Pecker-Wimel), C.: 1972b, Astron. Astrophys. 20, 87.

Harrison, B., Thorne, K. S., Wakano, M., and Wheeler, J. A.: 1965, Gravitation Theory and Gravitational Collapse, University of Chicago Press, Ill.

Larson, R. B. and Starrfield, S.: 1971, Astron. Astrophys. 13, 190.

Leung, V. C. and Wang, C. G.: 1971, Astrophys. J. 170, 499.

Lucy, L.: 1967, Astron. J. 72, 813.

Maran, S. P., Brandt, J. C., and Stecher, T. P.: 1971, The Gum Nebula and Related Problems, Goddard Space Flight Center Report X-683-71-375.

Mazarakis, M. and Stevens, W.: 1972, Astrophys. J. Letters 171, L97.

Oppenheimer, J. R. and Volkoff, G.: 1939, Phys. Rev. 55, 374.

Paczynski, B.: 1970, Acta Astron. 20, 47.

Paczynski, B.: 1972, Astrophys. Letters 11, 53.

Paczynski, B. and Ziolkowski, J.: 1967, Acta Astron. 17, 7.

Ramaty, R. and Boldt, E. A.: 1971, in S. P. Maran, J. C. Brandt, and T. P. Stecher (eds.), The

Gum Nebula and Related Problems, Goddard Space Flight Center Report X-683-71-375, p. 97.

Rose, W. K.: 1969, Astrophys. J. 155, 491.

Roxburgh, I. W.: 1967, Nature 215, 838.

Schwarzschild, M.: 1957, Structure and Evolution of the Stars, Dover Press, N.Y.

Tolman, R. C.: 1939, Phys. Rev. 56, 364.

Wheeler, J. C., Barkat, Z., and Buchler, J. R.: 1970, Astrophys. J. Letters 162, L129.

\section{DISCUSSION}

Van Horn: Is there any observational evidence that can be used to provide a direct estimate of the mass of the Gum nebula or the pre-supernova star?

Cohen: There are about $2 \times 10^{62}$ ionized particles in the Gum nebula and its size and age are about $460 \mathrm{pc}$ and $10^{4} \mathrm{yr}$ respectively. This distance can be traversed by $10 \mathrm{MeV}$ protons. If the energy released during neutron star formation is $3 \times 10^{53} \mathrm{erg}$ then the pre-supernova red-giant mass is $15 M_{\odot}$.

Wang: The minimum mass of a neutron star is accurately determined by the equation of state at subnuclear densities. The argument that for those neutron stars with mass less than $0.2 M_{\odot}$, their binding energy per nucleon would be less than the parent core of the degenerate matter, and therefore can not be formed, is a dangerous one. The dynamics of stellar collapse is far from being settled. I do not know if anyone has looked into the possibility of the formation of multiple collapsed objects, due to fission, for example, and their energetics may be shared.

Regarding the energetics of the Crab Nebula: we do not know the distance to the nebula to about a factor of two. The Crab Nebula can be roughly outlined as an ellipse. The maximum Doppler velocity at the line of sight to the Nebula is $1450 \mathrm{~km} \mathrm{~s}^{-1}$. Baade associated it with either the proper motion of the major or the minor axis with photographic plates taken ten years apart, he obtained 1.03 and $1.74 \mathrm{kpc}$ respectively. There are many other less direct arguments to estimate the distance. I think we should bear in mind the history of astronomer's estimate of distances.

With a very stiff equation of state at supernuclear densities, that is, connect between the limit of the usual nuclear physics calculation and the causality limit with a vertical line, you get the maximum mass of a neutron star at about $1.5 M_{\odot}$, the only way you can support a heavier neutron star is to make a stiff equation of state at the nuclear densities, and that region, unfortunately, is where we think we know some physics about nuclear matter. 